\title{
IT Evaluation of Foundation Healthcare Group Vanguard Project
}

\author{
Archana TAPURIA ${ }^{\mathrm{a}, 1}$, Maria KORDOWICZ ${ }^{\mathrm{b}}$, Mark ASHWORTH ${ }^{\mathrm{a}}$, Ewan FERLIE ${ }^{\mathrm{a}}$, \\ Vasa CURCIN ${ }^{a}$, Rositsa KOLEVA-KOLAROVA ${ }^{\text {a }}$, Julia FOX-RUSHBY ${ }^{\text {a }}$, Sylvia \\ EDWARDS $^{\mathrm{a}}$, Tessa CRILLYa and Charles WOLFE ${ }^{\mathrm{a}}$ \\ a King's College London, UK \\ ${ }^{\mathrm{b}}$ The University of Lincoln, UK
}

\begin{abstract}
The aim of the Foundation Healthcare Group (FHG) Vanguard model was to develop a sustainable local hospital model between two National Health Service (NHS) Trusts (a London Teaching Hospital Trust and a District General Hospital Trust) that makes best use of scarce resources and can be replicated across the NHS, UK. The aim of this study was to evaluate the provision, use and implementation of the IT infrastructure; based on qualitative interviews and focused mainly on the perspectives of the IT staff and the clinicians' perspectives. . In total 24 interview transcripts, along with 'Acute Care Collaboration' questionnaire responses, were analysed using a thematic framework for IT infrastructure, sharing themes across the vascular, paediatric and cardiovascular strands of the FHG programme. Findings indicated that Skype for Business had been an innovative and helpful development widely available to be used between the two Trusts. Clinicians initially reported lack of IT support and infrastructure expected at the outset for a national Vanguard project, but later appreciated that remote access to most clinical applications between the two Trusts became operational. The Local Care Record (LCR), an IT project was perceived to have been delivered successfully in South London. Shared technology reduced patient travelling time by providing locally based shared care. Spreading and scaling-up innovations from the Vanguard sites was the aspiration and challenge for system leaders.
\end{abstract}

Keywords. Vanguard, Foundation Healthcare Group, IT evaluation

\section{Introduction}

In January 2015, the National Health Service (NHS) invited individual organisations and partnerships to apply to become 'Vanguards' for the new care models programme, one of the first steps towards delivering the NHS Five Year Forward View [1]. The aim of the Foundation Healthcare Group (FHG) Vanguard programme was to develop a sustainable local hospital model that makes best use of scarce resources and can be replicated across the NHS [2]. The aspiration has been to enhance outcomes and access, improve cost-effectiveness, and meet the challenge of increased demand [3]. The FHG linked two hospital Trusts, a London Teaching Hospital Trust (LTHT) and a District General Hospital Trust (DGHT), who could work together more closely in a planned way to improve care without the formal organisational change of a merger or acquisition. The

\footnotetext{
${ }^{1}$ Corresponding Author, Dr. Archana Tapuria, School of Population Health and Environmental Sciences, King's College London, UK. Email: archana.tapuria@kcl.ac.uk
} 
context of the FHG model was within the Vanguard scheme developed by NHS England (NHSE) in which new models of care would be piloted and evaluated [4]. The setting of this model is innovative and of vital importance. The chosen LTHT is the largest hospital Trust in England, and a centre of world class expertise. The Vanguard attempted to link it with a small District General Hospital, and one which was struggling to provide specialist services like cardiology, neurology, etc. Patients therefore had to travel long distances to central London for care which otherwise could have been provided locally. The Vanguard provided IT links enabling some, but not all, services to be delivered locally. While it worked for some specialities, it didn't work for others and IT was the critical factor in influencing these outcomes. (Final FHG Report in Appendix). Thus, the role of IT in such a large-scale health and social care organizational change is crucial for the collaboration and execution of the project. The healthcare related IT evaluation was thus important and was based on qualitative interviews and was focussed mainly on the perspectives of the IT staff and clinicians across collaborative vascular, paediatric and cardiovascular care pathways.

\section{Methods}

Qualitative data analysis was used throughout the evaluation to distil complex data into themes which were used to capture the essence of observed and reported experience. Qualitative research findings presented here stem from analyses of interview data with staff, managerial and Board meetings, stakeholder events and documentary review. Interviews were semistructure, organised by question prompts, to elicit participant views of the Vanguard model. Six interviews were carried out for this IT evaluation with mid to senior-level IT, clinical and managerial Vanguard leads, all of whom are based at LTHT. 18 further interviews carried out as part of the clinical pathway evaluation stream included questions pertaining to IT support and infrastructure. An 'Acute care collaboration' questionnaire was completed by three senior expert IT leads. In total 24 interview transcripts, along with 'Acute Care Collaboration' questionnaire responses, were analysed using a six-phase analytic framework [5] to elicit inductively the significant features associated with IT infrastructure, clinical informatics and healthcare records sharing themes across the vascular, paediatrics and cardiovascular speciality areas. The six phases involved: 1-Transcription, 2-Familiarisation with the data, 3-Coding, 4Developing a working analytical framework, 5-Charting data into the framework matrix, and 6-Interpreting the data.

\section{Results}

The results are presented under various themes and sub-themes. (The details and quotes from the qualitative interviews are given in the Appendix)

\subsection{Clinician's perspective towards the provision, use and implementation of IT}

\subsection{1: Sharing of Patient records across the two Trusts}

Clinicians reported that they had to scan and load clinical documents and images manually, and that there was a lack of a suitable IT infrastructure and admin support for this purpose. This was time-consuming for the clinicians and a distraction from 
delivering patient care. They voiced their desire to be able to access each other's desktops to view letters, documents and images, across both sites to save time.

The electronic health record (EHR) and radiology systems between the two trusts appeared to lack interoperability and were unable to 'talk' to each other. The LTHT clinicians were able to view the scans at DGHT but DGHT clinicians were not able to view scans performed at LTHT and this issue had only been partially resolved by IT.

According to the clinicians, there were variations in the clinical systems and the clinical terminology used in EHRs between the two sites. Sharing clinicians' letters and patients' scans (Echo, MRI, CT, etc.) was perceived to be critical for quality holistic care provision. However, they did not feel sufficiently supported by an appropriate IT infrastructure for information sharing, and instead, had to upload patient documentation as email attachments or share via Skype screen sharing that was inadequate for patient care.

The clinicians found it problematic that their secretaries could access the systems but were unable to upload the documents/letters from the other Trust or print them and hence emails were sent in order to transfer information. According to the clinicians, if secretaries could preload required documents and scans, that would enable them to see more patients within the allocated time period for conducting remote clinics.

Those interviews conducted in the most recent quarter (Quarter 3, 2017/18) demonstrated a change in perception of IT functionality. Clinicians reported that remote access between DGHT and LTHT was operational and that they were able to access a range of clinical applications remotely, resolving most of the data sharing issues.

\subsection{2: Need for innovative IT solutions and provision of IT equipment:}

There was a perceived need for IT solutions to deliver more efficient communication pathways, inpatient transfers and remote data access. Sharing the electronic images/scans securely on iCloud was an option the Trusts had been exploring. 'Skype for Business' (SfB) was being used for communication between the two Trusts and for running the remote clinics. The clinicians report relatively high levels of turnover of Vanguard IT staff, which led to backlogs in resolving IT issues and that there was scope for further IT development.

Laptops were being used for virtual clinics, but their provision was viewed by participants as insufficient. Similarly, the process of upgrading Windows machines was reported as being slow. Occasional difficulties with Wifi access too were reported.

\subsection{IT team's perspective towards the provision and implementation of IT:}

The information around the following themes is derived from both interview data and the 'Acute care collaboration' questionnaire answered by three senior IT leads, whose perspectives add an additional layer of 'triangulation' to the evaluation findings. The feedback from IT leads suggests an awareness of the aforementioned issues 'on the ground'. Notably, the greatest reported challenge has been in establishing an effective IT workstream coordination and management capability.

\subsection{1: Use of technology for sharing of information:}

The following technology solutions were implemented for record sharing across the two Trusts. Furthermore, the Vanguard senior leadership team, in response to the evaluation team's (Qualter1, 2017)) formative feedback regarding IT provision, confirmed that LTHT was the first Trust in England to comprehensively roll out Windows 10 that was 
likely to provide a solution to many of the difficulties previously cited by interview participants.

a) SfB: Use of SfB facilitated communication between consultants on different sites to allow direct patient care to be discussed and secure sharing of patient information. The wider rollout of SfB to LTHT clinicians in the Cardiovascular pathway was dependent on a desktop (device) upgrade to Windows 10 which was implemented. SfB was used to conduct virtual clinics, consultant-to-consultant and consultant-to-patient consultations.

b) Remote Access: Remote access to LTHT and DGHT systems was fully tested, available and functioning and enabled two-way communication between LTHT and DGHT in order to facilitate access to clinical systems regardless of site base. Remote access uses standard Java and Citrix Remote Desktop solutions. Clinicians were able to use their existing credentials to gain access and view patient records through this secure link.

c) King's Health Partners Local Care Record (LCR): The aim of the Vanguard was to allow DGHT staff access to the King's Health Partners LCR system. The LCR is a readonly interoperability platform that allows patient healthcare record sharing between healthcare organisations including London Teaching Hospital NHS Trusts and more than 120 GP practices. A 'proof of concept' was run to inform the work which was required to implement the LCR at DGHT. The costs and resourcing required for specific projects, such as access to the LCR, were developed as individual proposals that addressed the use of secondments or short-term contract assignments, as necessary.

d) Picture Archiving and Communication System (PACS) Interconnection: The aim of the Vanguard was to interconnect PACS systems between the two Trusts to allow crosssite access to patient healthcare images for direct patient care. The interconnection of PACS was dependent on implementation of Sectra systems provided by a private software supplier at both Trusts, and the configuration of software and secure network access. Sectra PACS went live in May 2017 for both the Trusts. DGHT requested Cross Platform Workflow to be initiated in conjunction with DGHT's Sectra upgrade. Due to contractual obstacles, implementation was not feasible before programme end March 2018.

\subsubsection{Focusing on interoperability, information governance and data sharing:}

The pathway to full, real-time, interoperable systems was taken in steps that can be measured by achieving the 'maturity levels' 1-5; level 5 being full maturity. (Appendix) Data sharing for the purposes of direct patient care was covered by existing data sharing arrangements between Trusts.

\subsection{Barriers to implementation faced:}

Recruitment and retention of personnel to the programme had been very challenging, given the tight timeframes involved and fixed-term nature of assignments. Consultants expressed the need for continuous and strong IT leadership and coordination of IT systems across both the Trusts to exchange patient data was a challenge. Feedback from senior Vanguard programme management leads highlighted that although the Vanguard is a nationally funded programme, delivery is through local IT services that needed dedicated resources to deal with additional demand resulting from the programme. In terms of the financial requirements of an IT programme to support the Vanguard, an underspend of the allocated IT budget was forecasted. (Vanguard Programme IT Workstream Summary Progress Report, 30. Jan 18, v0.2 included in the Appendix). 


\section{Discussion and Conclusion}

The IT evaluation provided an overview of the challenges faced in exchanging patient data across the two Trusts and the implementation of the IT infrastructure required to achieve smooth collaboration between them. It also reported on the pilot projects and implementation of software tools and IT programmes planned to make the collaboration feasible. The salient features of the evaluation findings and its implications are: 1 . The overall impression was that clinicians initially reported they had not received the IT support and leadership expected at the outset for a national Vanguard project. Inefficiencies in clinical delivery occurred as a result of difficulties transferring data from DGHT to LTHT. Clinical activity could have been increased in Virtual clinics if data had been more accessible. Improvements were reported particularly in terms of remote access of EHRs and scans between DGHT and LTHT. 2. Skype for Business has been an innovative and helpful development. SfB has become widely available and used at both Trusts. Some participants reported a perceived lack of IT infrastructure. However, more recent Trust-wide Windows 10 rollout will support IT developments and should deliver further benefits for clinical teams. 3. Provision of laptops is the preferred hardware solution for conducting virtual clinics. Those who were allocated a new laptop reported in glowing terms, while those who were not, reported poor access to equipment, stressing the need for better laptop availability. 4. The way in which shared technology saved patients from travelling to LTHT and they ended up having shared care which was locally based, was seen as a successful outcome. 5. The LCR is an IT project that was perceived to have delivered successfully in south London. On reviewing LCR 'viewing statistics', it is clear that LCR has high levels of uptake and usability. Implementation at DGHT was a quick win with knock-on efficiencies. At the time, there was considerable IT inertia and scepticism about remote working. In the post-COVID-19 landscape, which brought with it unprecedented digital change, future work may include revisiting the IT connectivity issues that held back the linkage to see which are now working well. Spreading and scaling-up innovations from the Vanguard sites is the aspiration and challenge for system leaders. Further work is needed at the national level to remove legal, and financial barriers and make use of the learning and development of the IT infrastructure done during this FHG Vanguard project that can be applied across the other Vanguard sites too.

\section{References}

[1] https://www.england.nhs.uk/wp-content/uploads/2015/11/new_care_models.pdf Accessed on 2nd Jan 2021

[2] Foundation Healthcare Group value generation hypothesis tree; Value Assessment Framework submitted 8 February 2016

[3] https://www.guysandstthomas.nhs.uk/resources/innovation/Vanguard-End-of-Year-Report.pdf Accessed on 3rd Jan 2021

[4] https://www.england.nhs.uk/wp-content/uploads/2015/12/acc-uec-support-package.pdf Accessed on 2nd Jan 2021

[5] Fereday J, \& Muir-Cochrane E. Demonstrating rigor using thematic analysis: A hybrid approach of inductive and deductive coding and theme development. International Journal of Qualitative Methods. 2006;5(1). 